\title{
Telmisartan regresses left ventricular hypertrophy in caveolin-1-deficient mice
}

\author{
Marta H Krieger ${ }^{1,4}$, Annarita Di Lorenzo ${ }^{2,4}$, Christine Teutsch ${ }^{3}$, Katalin Kauser ${ }^{3}$ and William C Sessa ${ }^{2}$
}

The role of angiotensin II (Ang II) in promoting cardiac hypertrophy is well known; however, its role in a spontaneous model of hypertrophy in mice lacking the protein caveolin-1 (Cav-1 KO) has not been explored. In this study, WT and Cav-1 KO mice were treated with angiotensin receptor blocker (ARB), telmisartan (Telm), and cardiac function was assessed by echocardiography. Treatment of Cav-1 KO mice with Telm significantly improved cardiac function compared with age-matched vehicle-treated Cav-1 KO mice, whereas Telm did not affect cardiac function in WT mice. Both left ventricular (LV) weight to body weight ratios and LV to tibial length ratios were also reverted by Telm in Cav-1 KO but not in WT mice. LV hypertrophy was associated with increased expression of natriuretic peptides $\mathrm{A}$ and $\mathrm{B}, \beta$-myosin heavy chain and TGF- $\beta$, and Telm treatment normalized the expression of these genes. Telm reduced the expression of collagen genes (Col1 A and Col3A) and associated perivascular fibrosis in intramyocardial vessels in Cav-1 KO mice. In conclusion, Telm treatment reduces indexes of cardiac hypertrophy in this unique genetic model of spontaneous LV hypertrophy. Laboratory Investigation (2010) 90, 1573-1581; doi:10.1038/labinvest.2010.116; published online 28 June 2010

KEYWORDS: angiotensin; cardiac function; caveolin-1; hypertrophy

Several lines of experimental and clinical studies have identified a key role of the renin-angiotensin-aldosterone system (RAAS) in the pathophysiology of a number of cardiovascular diseases, such as myocardial infarction, stroke and congestive heart failure. In addition to angiotensin-converting enzyme (ACE) inhibitors, in the past few years, angiotensin II (Ang II) receptor blockers (ARBs) that selectively antagonize the Ang II type 1 receptor (AT1R), such as telmisartan (Telm), became a valid alternative approach to interfere with renin-angiotensin-aldosterone axis. ${ }^{1}$ Although ARBs selectively antagonize the actions of Ang II, there is still debate over the role of additional mechanisms by which they improve cardiac function, ${ }^{2}$ such as activation of PPAR- $\gamma^{3}$ or improving endothelial function. ${ }^{4}$

Caveolae are $50-100 \mathrm{~nm}$ invaginations of the plasma membrane that are decorated by caveolins (Cav) as their signature coat proteins. ${ }^{5,6}$ There are three Cav genes in mammals, Cav-1, Cav-2 and Cav-3, and genetic knockouts of Cav-1 and Cav-3 abolish the formation of caveolae in cells that express these isoforms, whereas the loss of Cav-2 does not qualitatively influence caveolae, but leads to pulmonary hyperplasia. ${ }^{7-11}$ In the heart, Cav-1 is highly expressed in the vascular endothelium and to a lesser extent in cardiac fibroblasts and smooth muscle, whereas Cav-3 is exclusively expressed in cardiac myocytes but not in vascular cells. ${ }^{12}$ Interestingly, deletion of either Cav-1 or Cav-3 results in cardiac hypertrophy, left ventricular (LV) dilation and dysfunction, and combined deletion of both genes results in severe cardiomyopathy, hypertrophy, disorganization and degeneration of the cardiac myocytes and interstitial fibrosis. ${ }^{13,14}$ In the hearts of Cav-1 KO mice, there is evidence for increased nitrosative stress and endothelial dysfunction because of enhanced endothelial nitric oxide synthase (eNOS) activation; these effects are suppressed by pharmacological blockade of eNOS or by the genetic reconstitution of Cav-1 back into the endothelium. ${ }^{12,15}$ However, the role of Ang II in promoting the cardiac phenotype in Cav-1 KO mice has not been examined. Thus, the aim of this study was to investigate the effect of the ARB, Telm, on functional and molecular markers of cardiac hypertrophy in Cav-1 KO mice.

\footnotetext{
${ }^{1}$ Department of Anatomy, Cell Biology, Physiology and Biophysics, Institute of Biology, State University of Campinas-UNICAMP, Campinas, São Paulo, Brazil; ${ }^{2}$ Vascular Biology and Therapeutics Program and Department of Pharmacology, Yale University School of Medicine, New Haven, CT, USA and ${ }^{3}$ Boehringer-Ingelheim, Ridgefield, CT, USA

Correspondence: Professor WC Sessa, PhD, Vascular Biology and Therapeutics Program, Yale University School of Medicine, Amistad Research Building, 10 Amistad Street, New Haven, CT 06519, USA.

${ }^{4}$ These authors contributed equally to this work.

Received 5 January 2010; revised 30 April 2010; accepted 12 May 2010
} 


\section{MATERIALS AND METHODS}

\section{Treatment of Cav-1 KO Animals}

Age-matched 8-week-old male wild-type (WT, C57Bl6) and congenic $\mathrm{Cav}_{-1}^{-1-}$ mice on a C57Bl6 background ${ }^{16}$ were treated with Telm (Boehringer-Ingelheim Pharmaceutical, Ridgefield, CT, USA) $(1 \mathrm{mg} / \mathrm{kg} /$ day in drinking water $)$ or a vehicle for 8 weeks. The mice were anesthetized with ketamine/xylazine $(100 \mathrm{mg} / \mathrm{kg}$ i.m.) and PBS perfusion of the right ventricle (RV) under physiological pressure $(\sim 20 \mathrm{~mm} \mathrm{Hg})$, and their hearts were dissected under ice and used for the experiments.

\section{Echocardiography Studies}

The cardiac dimensions of conscious mice were monitored using transthoracic echocardiography (Vevo 770 VisualSonics, Toronto, Canada), both before and after 8 weeks of treatment as described. ${ }^{17}$ In brief, LV M-mode (VisualSonics) was used and all measurements were made during 3-6 consecutive cardiac cycles, and the average values were used for analysis. LV end-diastolic (LVDd) and end-systolic (LVDs) dimensions, as well as the thickness of the interventricular septum (IVST) and posterior wall (PWT) were measured from the M-mode tracings, and fractional shortening (FS) was calculated as (LVDd-LVDs)/LVDd. Diastolic measurements were taken at the point of maximum cavity, and systolic measurements were made at the point of minimum dimension, using the leading-edge method.

\section{Morphometric Analysis}

Mice were killed and their tissues harvested. Tibias were dissected and measured. The heart was stopped at diastole $(\mathrm{KCl}, 14 \mathrm{~mm})$ and dissected to obtain $\mathrm{RV}$ separated from $\mathrm{LV}$ plus septum $(\mathrm{LV}+\mathrm{S})$ and were then weighed. For morphometric analysis, LV samples obtained from the free wall, at the level of papillary muscle, were fixed in $4 \%$ buffered formalin and embedded in paraffin, cut in $4 \mu \mathrm{m}$ sections and subsequently stained with hematoxylin and eosin. Two randomly selected sections from each animal were visualized by light microscopy using an objective with a calibrated magnification $(\times 400)$. Myocytes with visible nucleus and intact cellular membranes from areas of transversely cut muscle fibers were chosen for diameter determination. The width of individually isolated cardiomyocyte displayed on a viewing screen was manually traced, across the middle of the nuclei, with a digitizing pad and determined using a computerassisted image analysis system. Quantification of LV fibrosis involved Picrosirius red staining for detection of collagen in the heart. Sections measuring $6 \mu \mathrm{m}$ were deparaffinized, rehydrated and stained with Picrosirius red using a modification of the method. ${ }^{18}$ Images were obtained with a computerized digital camera, and collagen was examined under a microscope (Nikon E600) using polarized light. Cardiac myocyte width and ventricular fibrosis were also measured in the free LV wall using a computer-assisted morphometric system (Leica Quantimet 500, Cambridge,
UK). For each animal, approximately 10 visual fields were analyzed in a blinded manner.

\section{Quantitative Reverse Transcription PCR Analyses}

Samples from LV were homogenized with TRIzol reagent (Invitrogen). Total RNA was isolated with the RNAEasy kit (Qiagen) and transcribed into complementary DNA with the use of the TaqMan protocol (Applied Biosystem). Quantitative PCRs were carried out on an iCycler (Bio-Rad) with specific primers against the genes of interest, SYBRgreen (Bio-Rad) ready mix.

\section{Enzyme Immunosorbent Assay}

A commercially available EIA kit (Spi-Bio) was used to measure plasma levels of Ang II in WT and Cav-1 KO mice treated with vehicle or Telm for 8 weeks according to the manufacturer's instructions.

\section{Statistical Analysis}

The results of the experiments are expressed as the mean$s \pm$ s.e.m. A two-way analysis of variance was used to evaluate the data followed by a Tukey's post-test for comparison of the groups using Prism 3.0 software. A confidence level of $P<0.05$ was considered to be statistically significant.

\section{RESULTS}

\section{Telm Reverts Cardiac Hypertrophy in Cav-1 KO Mice}

To examine the contribution of Ang II to cardiac hypertrophy in Cav-1 KO mice, age-matched (8 weeks old), male WT (C57Bl6) and congenic Cav-1 $\mathrm{KO}$ mice on a $\mathrm{C} 57 \mathrm{Bl} 6$ background were treated with Telm $(1 \mathrm{mg} / \mathrm{kg}$ in drinking water) or vehicle for 8 -week duration. Cardiac dimensions were monitored in conscious mice using serial echocardiography (Vevo 770), before and after 8 weeks of treatment. As seen in Figures $1 \mathrm{a}-\mathrm{d}$ (black bars), compared with WT mice, Cav-1 KO mice had indices of increased concentric cardiac hypertrophy as determined by a reduction in LVDd and LVDs and an increase in IVSWT and PWT. However, there were no changes in FS as shown in Figure 1e, as the initial phase of pathological cardiac hypertrophy is likely to be a compensative adaptive response. ${ }^{1920}$ These data are consistent with previous reports documenting cardiac abnormalities and dysfunction in Cav-1 KO mice., ${ }^{9,13,14}$ Treatment of WT mice with Telm for 8 weeks had no appreciable effects on cardiac dimensions as monitored through echocardiography. However, treatment of Cav-1 KO mice with Telm increased LVDd and LVDs and reduced both PWT and IVSWT (Figure 1; Telm group). Improvements in cardiac dimensions were obvious through serial echocardiography (Figure 1f). Morphometric assessments of the myocyte hypertrophy in Telm-treated mice revealed that the Cav-1 KO cardiomyocytes were wider than control myocytes and Telm reduced myocyte width (Table 1). Cardiac morphometry showed that LV weight to body weight (BW) ratios and LV to tibial length (TL) ratios as well as RV weight to that of BW and RV to TL were also reduced by Telm in Cav-1 KO but not WT mice (Figures 2a-d). 

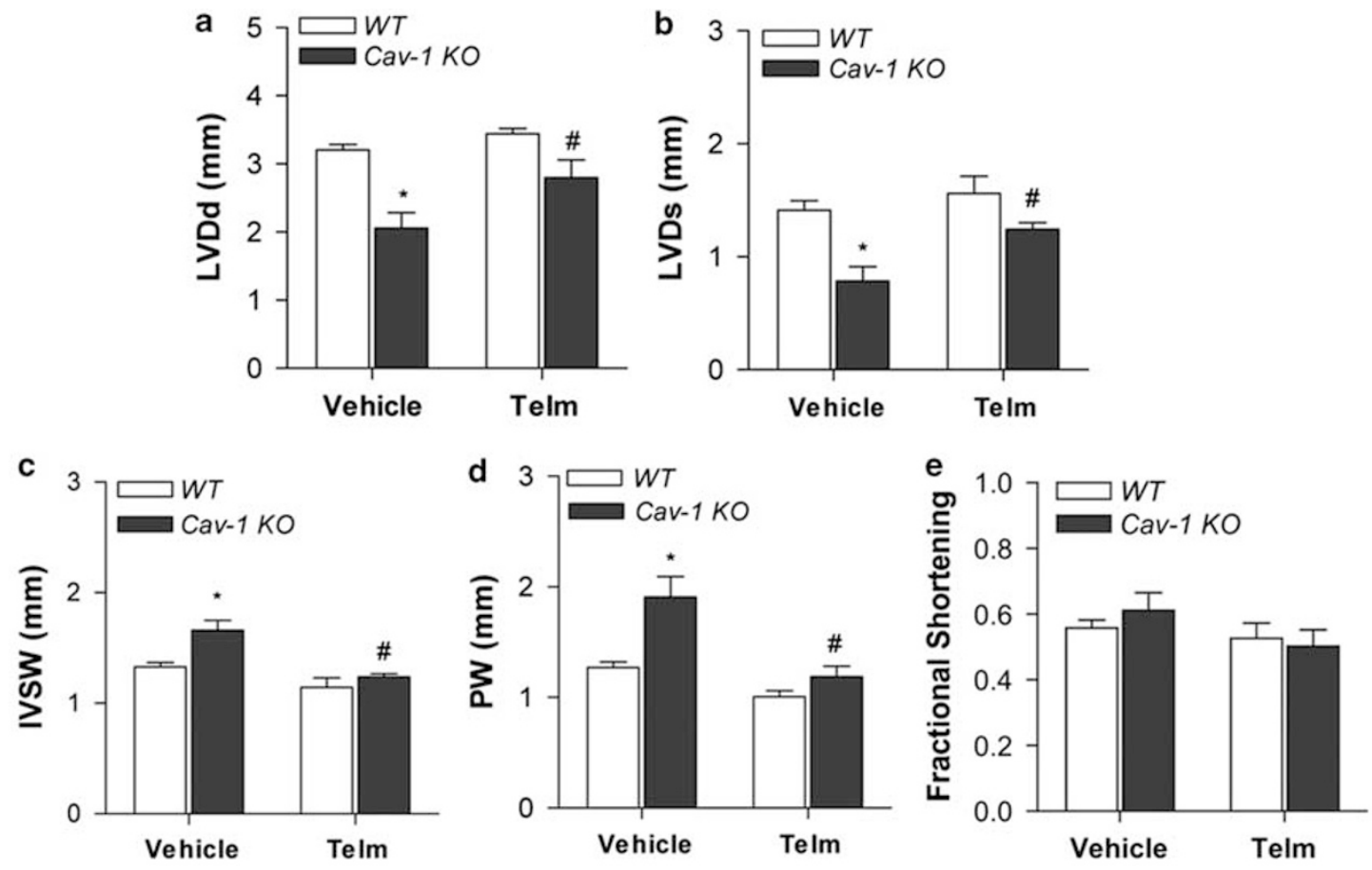

f

Vehicle

Telm
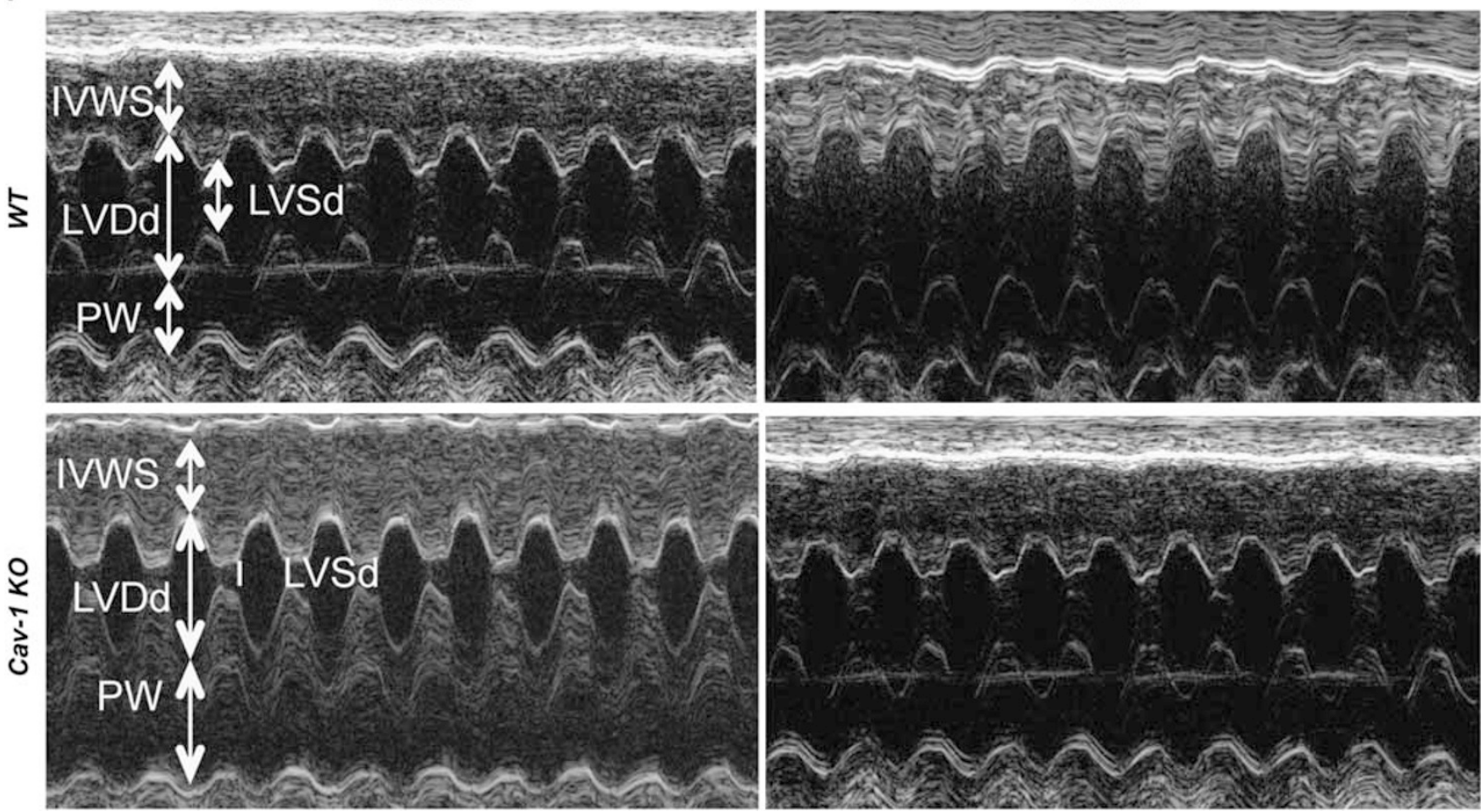

Figure 1 Telmisartan (Telm) reduces cardiac hypertrophy in Cav-1 KO mice. Serial echocardiography was performed in the four groups of mice as described and the following parameters (LVDd, LVDs, PW, IVSW and FS; a-e) were measured before and after treatment with Telm for 8 weeks. (f) Picture representative of ten individual records from post-1 $\mu \mathrm{M}$ Telm in comparison with vehicle treatment by transthoracic echocardiography on WT and Cav-1 KO mice. Data are mean \pm s.e.m. with $n=10$ for each group tested, ${ }^{*} p<0.05$.

Telm Normalizes the Expression of Genes Associated with LV Hypertrophy, Inflammation and Cardiac Fibrosis in Cav-1 KO Mice

We next examined gene expression of natriuretic peptides $\mathrm{A}$ and $-\mathrm{B}(\mathrm{Nppa}$ and $\mathrm{Nppb})$ and $\beta$-myosin heavy chain
( $\beta$-MHC) using total RNA isolated from LV of treated WT and Cav-1 KO mice by q-PCR. As seen in Figures $3 \mathrm{a}-\mathrm{c}$, the expression of all three hypertrophic markers were elevated in vehicle-treated Cav-1 KO mice compared with WT mice. Treatment with Telm reduced the expression of these genes to 
Table 1 Cardiomyocyte morphology and indices of fibrosis in WT and Cav-1 ${ }^{-1-}$ mice treated with vehicle or telmisartan

\begin{tabular}{lcccc}
\hline Group & WT+vehicle $(n=6)$ & WT+Telm $(n=6)$ & Cav-1 $^{-1-}+$ vehicle $(n=6)$ & Cav-1 ${ }^{-\prime-}+$ Telm $(n=6)$ \\
\hline Cardiomyocyte width $(\mu \mathrm{m})$ & $19 \pm 0.7$ & $18 \pm 0.5$ & $28 \pm 0.6^{*}$ & $20 \pm 0.7^{\#}$ \\
Collagen interstitial area (\%) & $2.8 \pm 0.25$ & $2.9 \pm 0.10$ & $3.0 \pm 0.27$ & $2.9 \pm 0.32$ \\
Collagen perivascular area (\%) & $1.4 \pm 0.1$ & $1.6 \pm 0.1$ & $4.0 \pm 0.05^{*}$ & $1.5 \pm 0.07^{\#}$ \\
\hline
\end{tabular}

Left ventricular cardiomyocyte width $(\mu \mathrm{m})$, left ventricular collagen deposition in cardiac interstitium and perivascular regions from the four different groups of mice in the study.

Data are mean \pm s.e.m., $n=6$ mice per group.

${ }^{*} P<0.05$ compared with WT mice.

${ }^{\#} P<0.05$ compared with $\mathrm{Cav}-1^{-1-}$.
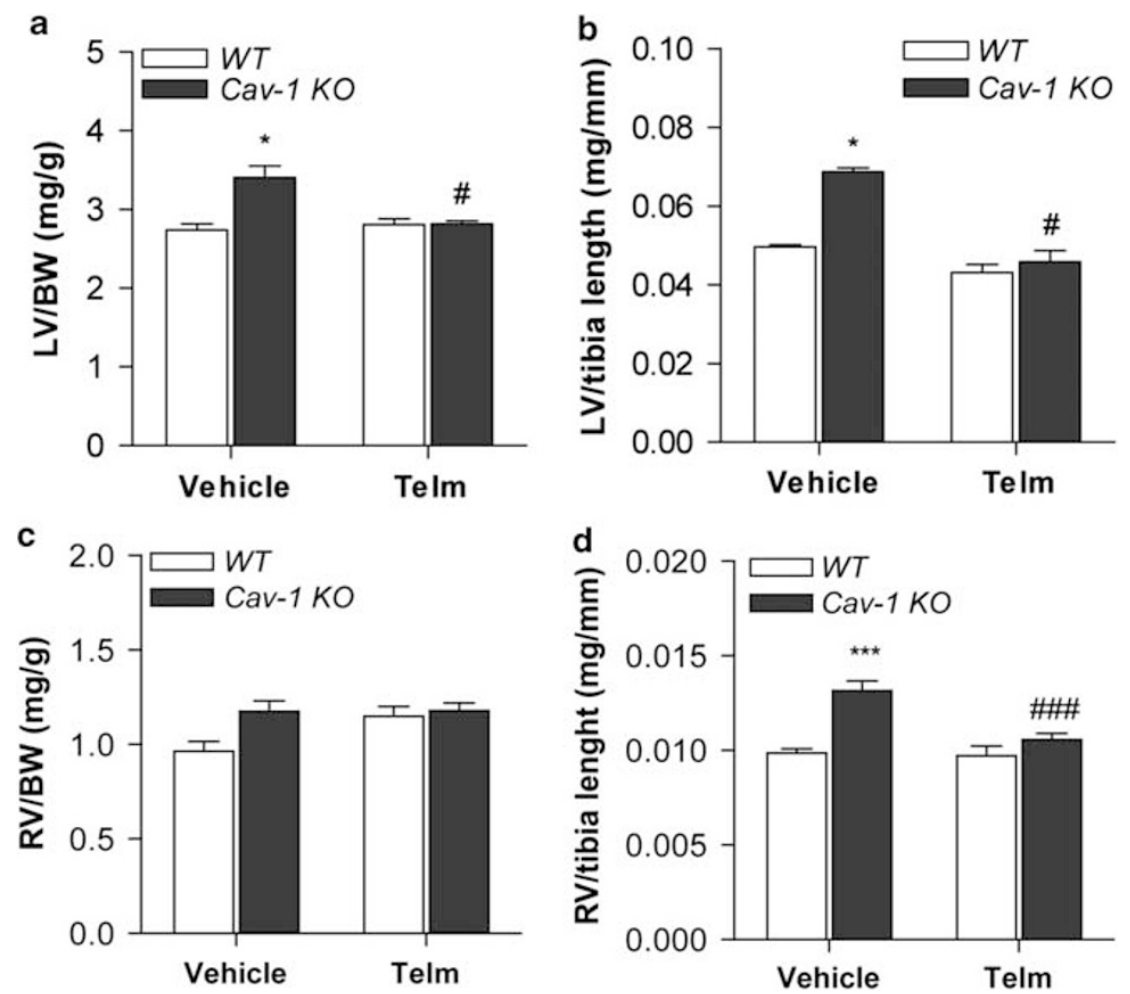

Figure 2 Telm improves cardiac morphometry in Cav-1 KO mice. WT and Cav-1 KO mice were treated with Telm as above and after killing cardiac morphometrics assessed. (a) Left ventricular weight (LV)/body weight (BW) in $\mathrm{mg} / \mathrm{g}$; (b) LV/tibia length in $\mathrm{mg} / \mathrm{cm}$; (c) right ventricular (RV) per BW in $\mathrm{mg} / \mathrm{g}$; and (d) RV/tibia length in $\mathrm{mg} / \mathrm{cm} . \mathrm{N}=10$ mice for each group tested, ${ }^{*} P<0.05$.

levels observed in vehicle-treated or Telm-treated WT mice. As there is evidence that cardiac chemo/cytokines may participate in hypertrophy, we assessed the mRNA levels of macrophage chemotactic peptide-1 (MCP-1) in vehicle- and Telm-treated mice. As seen in Figure 3d, vehicle-treated Cav$1 \mathrm{KO}$ mice had elevated levels of MCP-1 compared with WT mice and treatment of Cav-1 KO mice reduced MCP-1 gene expression. It has been reported that p42/44 MAP kinase cascade is hyperactivated in Cav-1 KO cardiac tissue and in isolated cardiac fibroblasts, and this effect contributes to the cardiac hypertrophy. ${ }^{13}$ Telm treatment did not affect the increase in MAPK phosphorylation and eNOS expression observed in the LV of Cav-1 KO compared with WT mice (Figure 3e).

There is evidence that Cav-1 KO mice have increased cardiac and perivascular fibrosis because of increased TGF- $\beta$ mediated signaling. As seen in Figures $4 \mathrm{a}-\mathrm{c}$, Cav- $1 \mathrm{KO}$ mice had increased expression of TGF- $\beta$ and the interstitial collagen $\alpha$ chains, CollA and Col3A. Treatment with Telm reduced both Col1A and Col3A gene expression, but did not influence TGF- $\beta$ levels. The reduction in gene expression was associated with less interstitial collagen and perivascular fibrosis (through Picrosirius red) in intramyocardial vessels in Telm-treated mice (Table 1 and Figure 4d). 

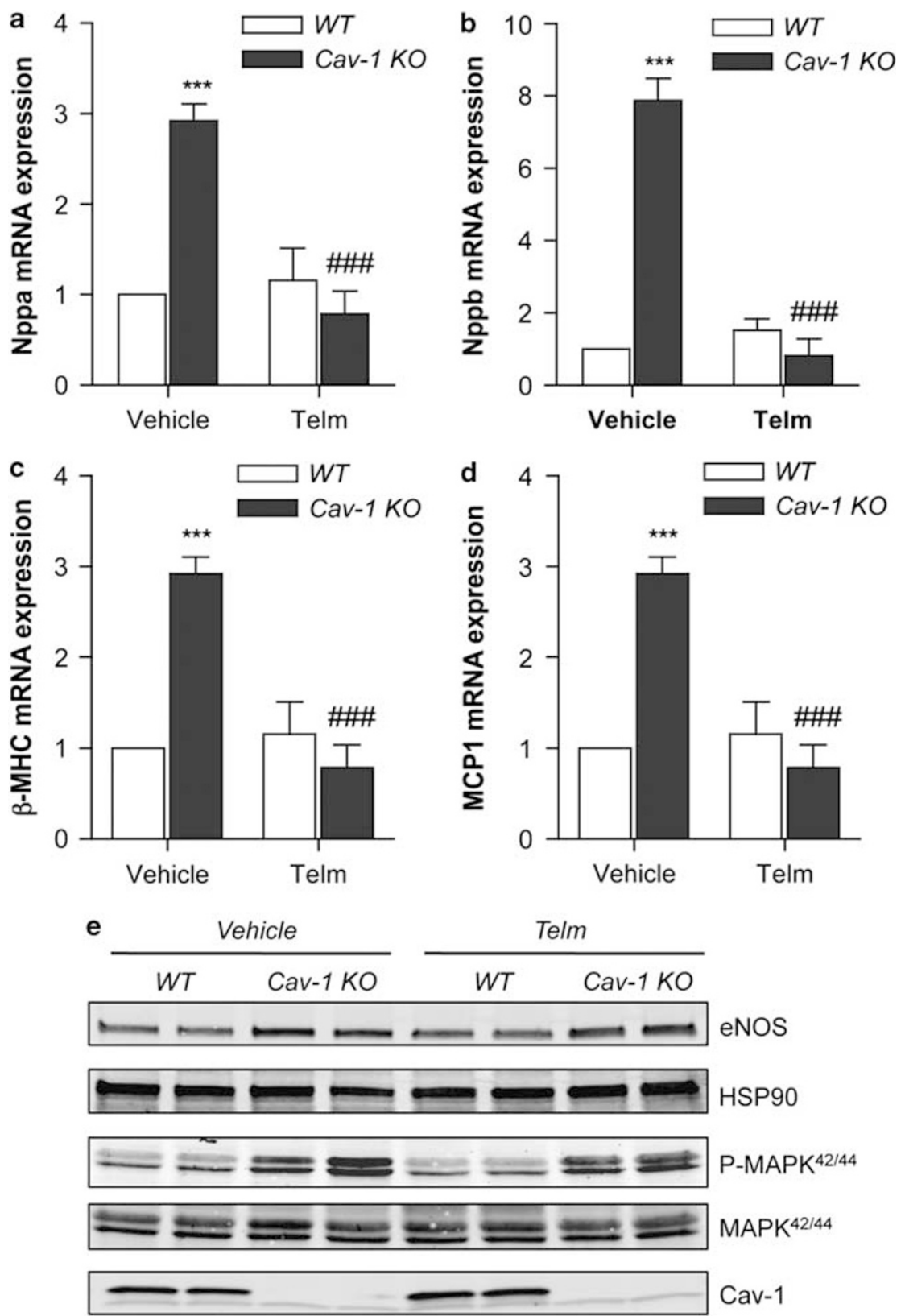

Figure 3 Telmisartan reduces hypertrophic and inflammatory gene expression in Cav-1 KO mice. (a-c) Pro-natriuretic peptide type b and a (Nppb and $\mathrm{Nppa}$ ) and $\beta$ - myosin heavy chain ( $\beta$ - MHC) genes were measured. (d) Monocyte chemoattractant protein-1 (MCP-1) expression was quantified in the four groups. (e) Western blot analysis was performed in different treatment group for eNOS, P-MAPK ${ }^{42 / 44}$ and MAPK ${ }^{42 / 44}$, HSP90 and Cav-1. N $=6$ mice for each group tested, ${ }^{*} P<0.05$.

Telm Increases the Cardiac Expression of Angiotensin Receptor 2 (AT2R) and Reduces the Plasma Levels of Ang II but does not Affect Other Components of the Renin-angiotensin System in Cav-1 KO Hearts

To examine whether hypertrophic, Cav-1 KO mice showed altered expression of components of the cardiac renin-angiotensin system or whether Telm influenced gene expression, we quantified the expression of AT1, AT2, renin, angiotensinogen (angiogen) and ACE in the hearts from treated WT and Cav-1 KO mice. As seen in Figure 5, no intrinsic differences in gene expression were found in vehicle- or Telmtreated WT and Cav-1 KO mice except that Telm significantly increased the levels of AT2R in the hearts of Cav-1 KO mice. However, measurement of blood levels of Ang II revealed increased levels of Ang II in Cav-1 KO mice versus WT $(4.51 \pm 0.91$ and $0.45 \pm 0.07 \mathrm{fmol} / \mathrm{ml}$, respectively, $n=8$ mice per group). Treatment with Telm reduced the blood level of Ang II in Cav-1 KO mice but did not influence Ang II levels in WT mice $(1.24 \pm 0.26$ and $1.11 \pm 0.15 \mathrm{fmol} / \mathrm{ml}$, respectively). 

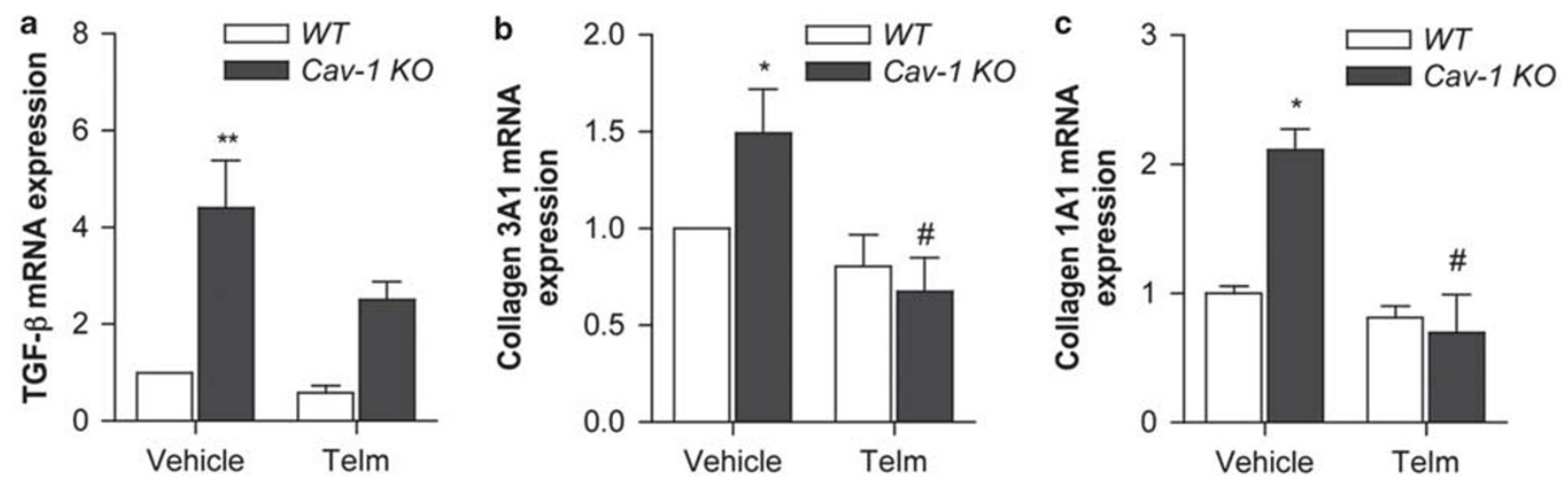

d

Vehicle
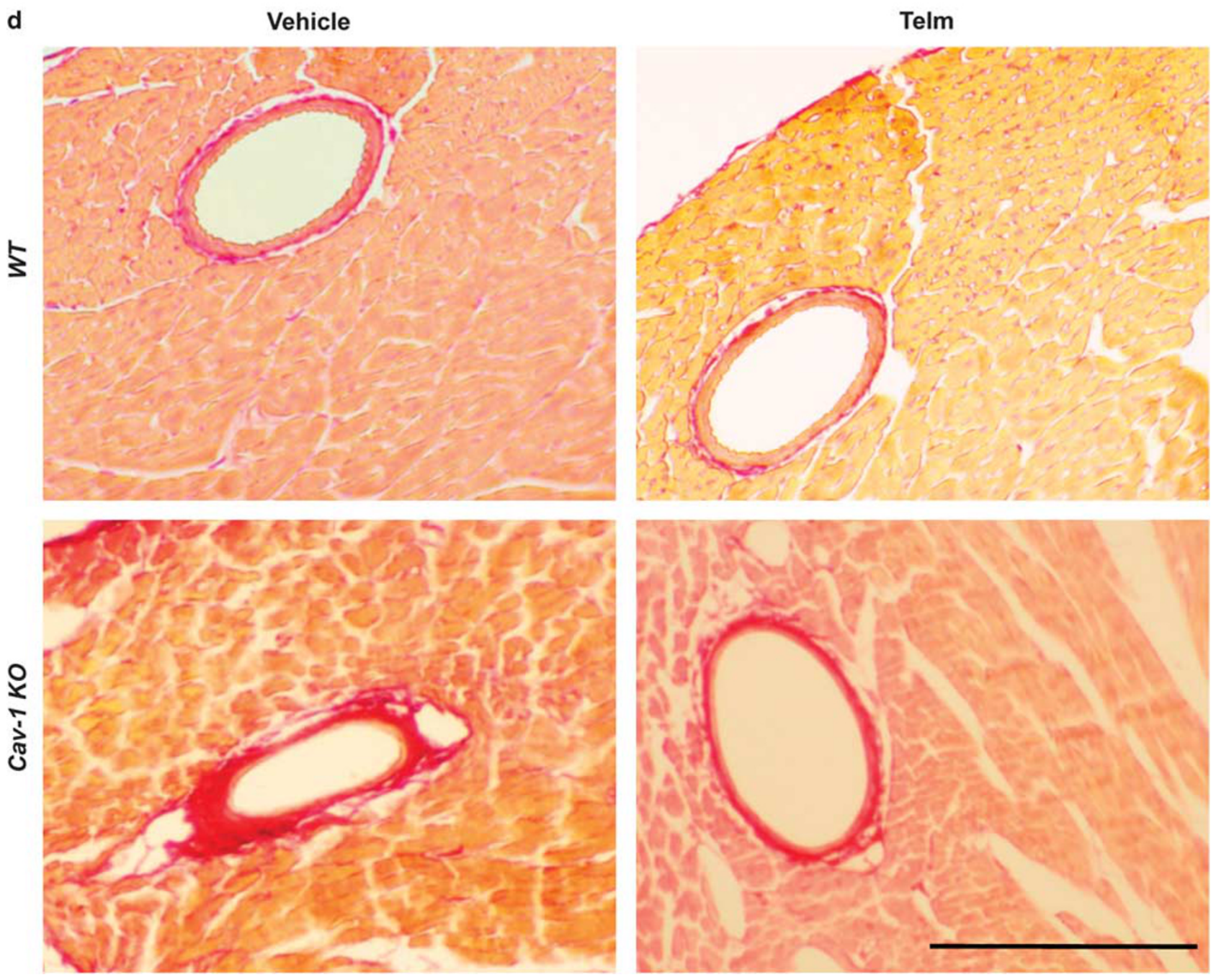

Figure 4 Telmisartan diminishes pro-fibrotic gene expression and perivascular collagen deposition in Cav-1 KO mice. (a) Transforming growth factor $\beta$ (TGF- $\beta$ ), (b) collagen 1A (Col1A) and (c) collagen 3A (Col3A) genes were quantified in the different treatment groups. (d) Photomicrographs of Picrosirius red-stained sections of heart section from different treatments containing perivascular collagen. The bar represents $100 \mu \mathrm{m}$.

\section{DISCUSSION}

The central finding of this study is that the ARB, Telm, improves the parameters of cardiac hypertrophy in Cav-1 KO mice, suggesting that the endogenous generation of Ang II contributes to cardiac hypertrophy in this model. Treatment with the AT1-receptor blocker Telm suppressed and reverted this abnormal remodeling, as evidenced by serial echocardiography, as well as by a reduction in myocyte 

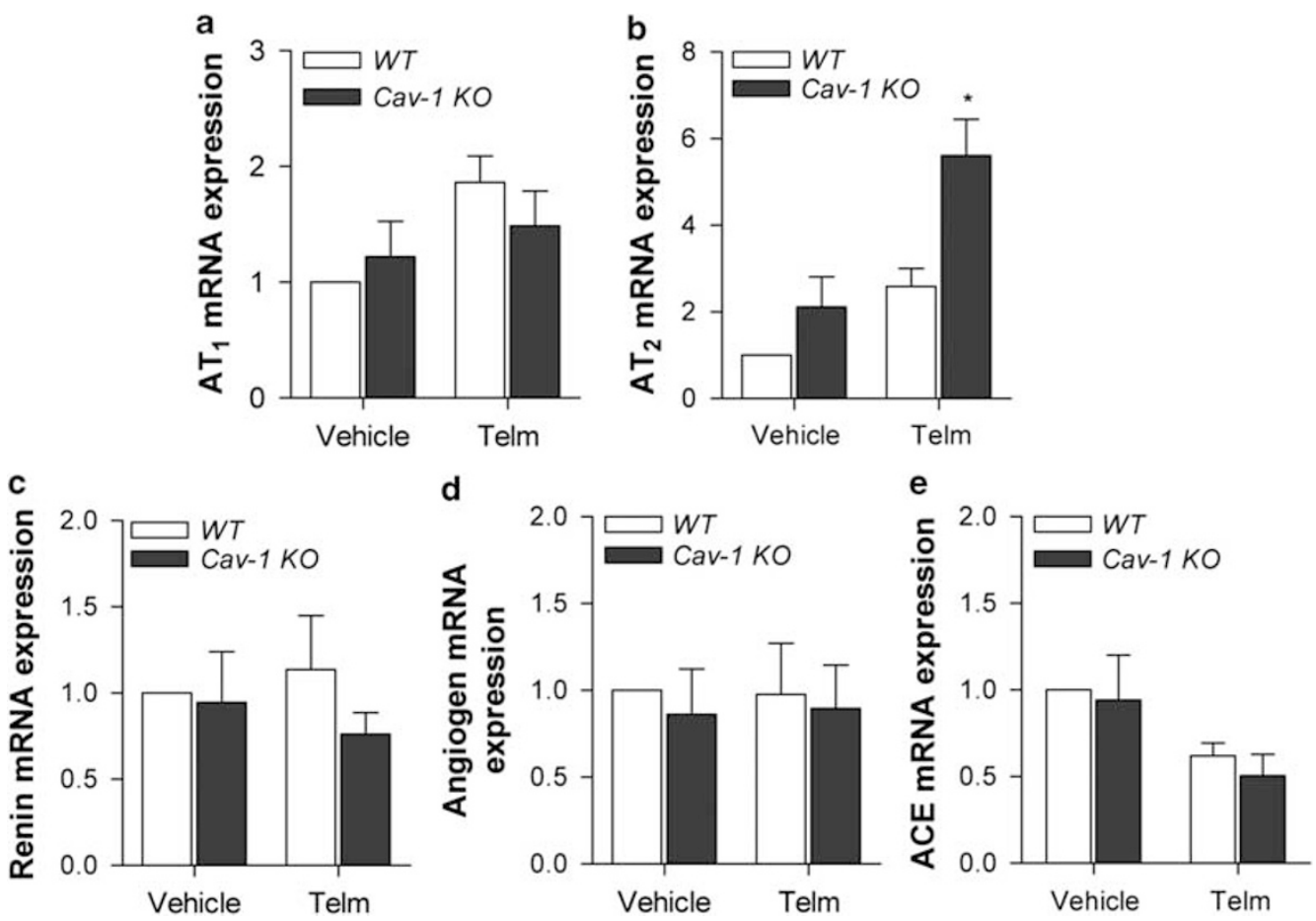

Figure 5 Telmisartan (Telm) increases the expression of ATR2 in Cav-1 KO mice. ATR1, ATR2, renin, angiotensinogen (angiogen) and angiotensin-converting enzyme (ACE) mRNA levels were measured in the left ventricle of vehicle- and Telm-treated mice $(\mathbf{a}-\mathbf{e}) . N=6$ for each group of mice tested, ${ }^{\star} P<0.05$.

dimensions, RV and LV mass. The reversal of cardiac remodeling in Cav-1 KO mice with Telm treatment likely occurs through several mechanisms, including improvement in endothelial function, reduction in inflammatory gene expression, fibrosis and blood Ang II levels and the upregulation of AT2 expression.

There are several papers showing that the genetic deletion of Cav-1 leads to spontaneous cardiac hypertrophy. This is surprising, given that in the heart, Cav-1 is primarily found in the vasculature (endothelium and smooth muscle) and cardiac fibroblasts, but not in cardiac myocytes, which express ample amounts of Cav-3. Thus, the loss of Cav- 1 in vasculature and cardiac fibroblasts is sufficient to promote abnormal cardiac remodeling. Interestingly, re-expression of Cav-1 into the endothelium of global Cav-1 KO mice rescues cardiac hypertrophy, suggesting that Cav-1 in the endothelium is critical for this phenotype. ${ }^{21}$ With this in mind, what is the potential endothelial-dependent mechanism linking Cav-1 with hypertrophic signaling? It is well appreciated that Cav-1 serves as an inhibitory clamp on eNOS function, ${ }^{22,23}$ and the loss of Cav-1 increases basal eNOS activity, $\mathrm{NO}$ release and superoxide generation and promotes nitrosative stress during conditions of inflammation. Indeed, treatment of Cav-1 KO mice with NOS inhibitors reduces cardiac hypertrophy and improves exercise tolerance in them, ${ }^{15,24,25}$ and breeding of Cav-1 KO mice with eNOS-KO mice reverses impaired pulmonary function in them. ${ }^{26}$ Thus, dysregulated eNOS can explain, in part, the endotheliumdependent mechanism contributing to increased cardiac hypertrophy in Cav-1 KO mice. In addition, there are data suggesting that Cav- 1 negatively regulates TGF- $\beta$ signaling in cardiac fibroblasts, and the loss of Cav-1 enhances TGF- $\beta$ signaling leading to hypertrophy and tissue fibrosis. ${ }^{13,27,28}$ Studies in cardiac fibroblasts have also shown that Ang II increases reactive oxygen species and collagen synthesis, effects abolished by Telm, but not by the specific AT2receptor antagonist $\mathrm{P}-186 .{ }^{29,30}$ Therefore, in this study, the beneficial effect of Telm may be because of an improvement in endothelial function and/or suppression of cardiac remodeling by antagonizing AT1R in fibroblasts and reducing the circulating levels of Ang II.

Previous clinical and experimental studies have shown the beneficial effects of ARBs in the suppression of cardiac remodeling. However, these studies have shown that low doses of ARB can exert cardioprotective effects independent of blood pressure reduction. As the dosage used in the present study $(1 \mathrm{mg} / \mathrm{kg} /$ day in drinking water $)$ does not lower blood pressure in normotensive mice, ${ }^{31}$ it seems that the cardioprotective effect is independent of blood pressure. Such blood pressure-independent actions of Telm, mediating the transition from hypertrophy to heart failure, has been shown previously. ${ }^{32,33}$

The systemic and local RAAS has a key role in the compensatory neurohumoral response to myocardial injury. Advances in the molecular pharmacology of the AT1 receptor have led to the development of highly specific AT1R-specific $\mathrm{ARBs}$, and these compounds clearly block AT1R and reduce cardiac fibroblast proliferation and net accumulation of 
fibrillar collagen, both in vitro and in vivo. ${ }^{34}$ In this study, there were no differences in the local RAS in Cav-1 KO mice compared with WT mice, and Telm treatment did not influence AT1R, renin, angiotensinogen or ACE mRNA levels but significantly increased AT2R gene expression in Cav-1 $\mathrm{KO}$ mice. This effect was associated with an increase in circulating levels of Ang II in Cav-1 KO, reduced upon Telm treatment. How this occurs is not known but is consistent with the protective role of AT2R upregulation after Telm treatment. ${ }^{35,36}$ AT2R signaling has been implicated in the suppression of myocardial hypertrophy, ${ }^{34}$ fibroblast proliferation, ${ }^{37}$ and vascular cell hyperplasia, ${ }^{38}$ although little is known about the mechanisms. Previous studies have suggested the possible involvement of the bradykinin/nitric oxide (NO)/cGMP system ${ }^{39}$ in the AT2R-mediation of the prevention of heart failure after myocardial infarction, ${ }^{40}$ and the hypertrophic heart caused by aortic coarctation. ${ }^{8}$ Despite tremendous research efforts, the specific mechanisms of these protective actions, as well as the underlying role of the local RAS in myocardial remodeling remain enigmatic.

In summary, Telm reverses cardiac hypertrophy initiated by the loss of Cav-1. As Cav-1KO mice are normo- or hypotensive, the reversal of hypertrophy occurs independent of blood pressure and is likely because of an effect of the ARB on endothelial function and/or cardiac fibroblasts. Mechanistically, Telm reduced cardiac hypertrophy, normalized hypertrophic gene expression and reduced indices of cardiac fibrosis. Thus, it is likely that endogenous activation of AT1 receptors contributes to cardiac hypertrophy initiated by the genetic loss of Cav-1.

\section{ACKNOWLEDGEMENT}

This work was supported by grants R01 HL64793, R01 HL61371, R01 HL081190 and P01 HI70295 from the National Institutes of Health and a Sponsored Research Agreement from Boehringer-Ingelheim.

\section{DISCLOSURE/CONFLICT OF INTEREST}

The authors declare no conflict of interest.

1. Battershill AJ, Scott LJ. Telmisartan: a review of its use in the management of hypertension. Drugs 2006;66:51-83.

2. Rizos CV, Elisaf MS, Liberopoulos EN. Are the pleiotropic effects of telmisartan clinically relevant? Curr Pharm Des 2009;15:2815-2832.

3. Kappert K, Tsuprykov O, Kaufmann J, et al. Chronic treatment with losartan results in sufficient serum levels of the metabolite EXP3179 for PPARgamma activation. Hypertension 2009;54:738-743.

4. Watanabe T, Suzuki J, Yamawaki H, et al. Losartan metabolite EXP3179 activates Akt and endothelial nitric oxide synthase via vascular endothelial growth factor receptor-2 in endothelial cells: angiotensin II type 1 receptor-independent effects of EXP3179. Circulation 2005;112: 1798-1805.

5. Gratton JP, Bernatchez P, Sessa WC. Caveolae and caveolins in the cardiovascular system. Circ Res 2004;94:1408-1417.

6. Williams TM, Lisanti MP. The Caveolin genes: from cell biology to medicine. Ann Med 2004;36:584-595.

7. Razani $B$, Engelman JA, Wang XB, et al. Caveolin-1 null mice are viable but show evidence of hyperproliferative and vascular abnormalities. J Biol Chem 2001;276:38121-38138.

8. Wollert KC, Studer R, Doerfer K, et al. Differential effects of kinins on cardiomyocyte hypertrophy and interstitial collagen matrix in the surviving myocardium after myocardial infarction in the rat. Circulation 1997;95:1910-1917.

9. Zhao YY, Liu Y, Stan RV, et al. Defects in caveolin-1 cause dilated cardiomyopathy and pulmonary hypertension in knockout mice. Proc Natl Acad Sci USA 2002;99:11375-11380.

10. Razani B, Wang XB, Engelman JA, et al. Caveolin-2-deficient mice show evidence of severe pulmonary dysfunction without disruption of caveolae. Mol Cell Biol 2002;22:2329-2344.

11. Drab $M$, Verkade $P$, Elger $M$, et al. Loss of caveolae, vascular dysfunction, and pulmonary defects in caveolin-1 gene-disrupted mice. Science (New York, NY) 2001;293:2449-2452.

12. Murata $\mathrm{T}$, Lin Ml, Huang $\mathrm{Y}$, et al. Reexpression of caveolin-1 in endothelium rescues the vascular, cardiac, and pulmonary defects in global caveolin-1 knockout mice. J Exp Med 2007;204:2373-2382.

13. Cohen AW, Park DS, Woodman SE, et al. Caveolin-1 null mice develop cardiac hypertrophy with hyperactivation of p42/44 MAP kinase in cardiac fibroblasts. Am J Physiol 2003;284:C457-C474.

14. Park DS, Woodman SE, Schubert W, et al. Caveolin-1/3 doubleknockout mice are viable, but lack both muscle and non-muscle caveolae, and develop a severe cardiomyopathic phenotype. Am J Pathol 2002;160:2207-2217.

15. Wunderlich C, Schober K, Lange SA, et al. Disruption of caveolin-1 leads to enhanced nitrosative stress and severe systolic and diastolic heart failure. Biochem Biophys Res Commun 2006;340:702-708.

16. Yu J, Bergaya S, Murata $\mathrm{T}$, et al. Direct evidence for the role of caveolin-1 and caveolae in mechanotransduction and remodeling of blood vessels. J Clin Invest 2006;116:1284-1291.

17. Fernandez-Hernando $C$, Jozsef $L$, Jenkins $D$, et al. Absence of Akt1 reduces vascular smooth muscle cell migration and survival and induces features of plaque vulnerability and cardiac dysfunction during atherosclerosis. Arterioscler Thromb Vasc Biol 2009;29:2033-2040.

18. Sweat F, Puchtler H, Rosenthal Sl. Sirius Red F3ba as a stain for connective tissue. Arch Pathol 1964;78:69-72.

19. Frey N, Katus HA, Olson EN, et al. Hypertrophy of the heart: a new therapeutic target? Circulation 2004;109:1580-1589.

20. Frey N, Olson EN. Cardiac hypertrophy: the good, the bad, and the ugly. Annu Rev Physiol 2003;65:45-79.

21. Murata T, Lin Ml, Stan RV, et al. Genetic evidence supporting caveolae microdomain regulation of calcium entry in endothelial cells. J Biol Chem 2007;282:16631-16643.

22. Garcia-Cardena G, Martasek P, Masters BS, et al. Dissecting the interaction between nitric oxide synthase (NOS) and caveolin. Functional significance of the nos caveolin binding domain in vivo. J Biol Chem 1997;272:25437-25440.

23. Michel JB, Feron O, Sacks D, et al. Reciprocal regulation of endothelial nitric-oxide synthase by $\mathrm{Ca2+-calmodulin}$ and caveolin. J Biol Chem 1997;272:15583-15586.

24. Wunderlich $C$, Schober $K$, Kasper $M$, et al. Nitric oxide synthases are crucially involved in the development of the severe cardiomyopathy of caveolin-1 knockout mice. Biochem Biophys Res Commun 2008;377:769-774.

25. Wunderlich C, Schober K, Schmeisser A, et al. The adverse cardiopulmonary phenotype of caveolin-1 deficient mice is mediated by a dysfunctional endothelium. J Mol Cell Cardiol 2008;44:938-947.

26. Zhao YY, Zhao YD, Mirza MK, et al. Persistent eNOS activation secondary to caveolin-1 deficiency induces pulmonary hypertension in mice and humans through PKG nitration. J Clin Invest 2009; 119:2009-2018.

27. Del Galdo F, Lisanti MP, Jimenez SA. Caveolin-1, transforming growth factor-beta receptor internalization, and the pathogenesis of systemic sclerosis. Curr Opin Rheumatol 2008;20:713-719.

28. Sotgia F, Del Galdo F, Casimiro MC, et al. Caveolin-1-/- null mammary stromal fibroblasts share characteristics with human breast cancerassociated fibroblasts. Am J Pathol 2009;174:746-761.

29. Lijnen PJ, Petrov VV, Fagard RH. Angiotensin II-induced stimulation of collagen secretion and production in cardiac fibroblasts is mediated via angiotensin || subtype 1 receptors. J Renin Angiotensin Aldosterone Syst 2001;2:117-122.

30. Lijnen PJ, Petrov VV, Jackson KC, et al. Effect of telmisartan on angiotensin II-mediated collagen gel contraction by adult rat cardiac fibroblasts. J Cardiovasc Pharmacol 2001;38:39-48.

31. Takaya T, Kawashima S, Shinohara M, et al. Angiotensin II type 1 receptor blocker telmisartan suppresses superoxide production and 
reduces atherosclerotic lesion formation in apolipoprotein E-deficient mice. Atherosclerosis 2006;186:402-410.

32. Kobayashi N, Ohno T, Yoshida K, et al. Cardioprotective mechanism of telmisartan via PPAR-gamma-eNOS pathway in dahl salt-sensitive hypertensive rats. Am J Hypertens 2008;21:576-581.

33. Takenaka $\mathrm{H}$, Kihara $\mathrm{Y}$, Iwanaga $\mathrm{Y}$, et al. Angiotensin II, oxidative stress, and extracellular matrix degradation during transition to LV failure in rats with hypertension. J Mol Cell Cardiol 2006;41:989-997.

34. Bartunek J, Weinberg EO, Tajima M, et al. Angiotensin II type 2 receptor blockade amplifies the early signals of cardiac growth response to angiotensin II in hypertrophied hearts. Circulation 1999;99:22-25.

35. Liang B, Leenen FH. Prevention of salt-induced hypertension and fibrosis by AT1-receptor blockers in Dahl $S$ rats. J Cardiovasc Pharmacol 2008;51:457-466.

36. Li H, Gao Y, Grobe JL, et al. Potentiation of the antihypertensive action of losartan by peripheral overexpression of the ANG II type 2 receptor. Am J Physiol Heart Circ Physiol 2007;292: $\mathrm{H} 727-\mathrm{H} 735$.

37. Tsutsumi $\mathrm{Y}$, Matsubara $\mathrm{H}$, Ohkubo $\mathrm{N}$, et al. Angiotensin II type 2 receptor is upregulated in human heart with interstitial fibrosis, and cardiac fibroblasts are the major cell type for its expression. Circ Res 1998;83:1035-1046.

38. Stoll M, Steckelings UM, Paul M, et al. The angiotensin AT2-receptor mediates inhibition of cell proliferation in coronary endothelial cells. J Clin Invest 1995;95:651-657.

39. Tsutsumi Y, Matsubara H, Masaki H, et al. Angiotensin II type 2 receptor overexpression activates the vascular kinin system and causes vasodilation. J Clin Invest 1999;104:925-935.

40. Liu YH, Yang XP, Sharov VG, et al. Effects of angiotensin-converting enzyme inhibitors and angiotensin II type 1 receptor antagonists in rats with heart failure. Role of kinins and angiotensin II type 2 receptors. J Clin Invest 1997;99:1926-1935. 